\title{
Stenting of stenosed aortopulmonary collaterals: a new approach to palliation in pulmonary atresia with multifocal aortopulmonary blood supply
}

\author{
Karen A McLeod, Michael E Blackburn, John L Gibbs
}

\begin{abstract}
A patient with complex pulmonary atresia and increasing cyanosis due to stenoses of aortopulmonary collateral vessels improved after implantation of balloon expandable stents into the stenosed vessels. Stent implantation may provide an alternative to surgical palliation in this group of patients.
\end{abstract}

(Br Heart f 1994;71:487-489)

Patients with pulmonary atresia, ventricular septal defect, and multifocal pulmonary blood supply who survive into adulthood eventually develop increasing cyanosis because of pulmonary vascular disease or because of progressive stenosis of aortopulmonary collateral vessels. In patients who develop stenosis of collateral vessels aortopulmonary shunt surgery may provide successful short term palliation, ${ }^{1}$ but it may be difficult to identify a pulmonary artery of sufficient calibre to allow a successful shunt and thoracotomy in itself may increase the operative risk of later cardiopulmonary transplantation.

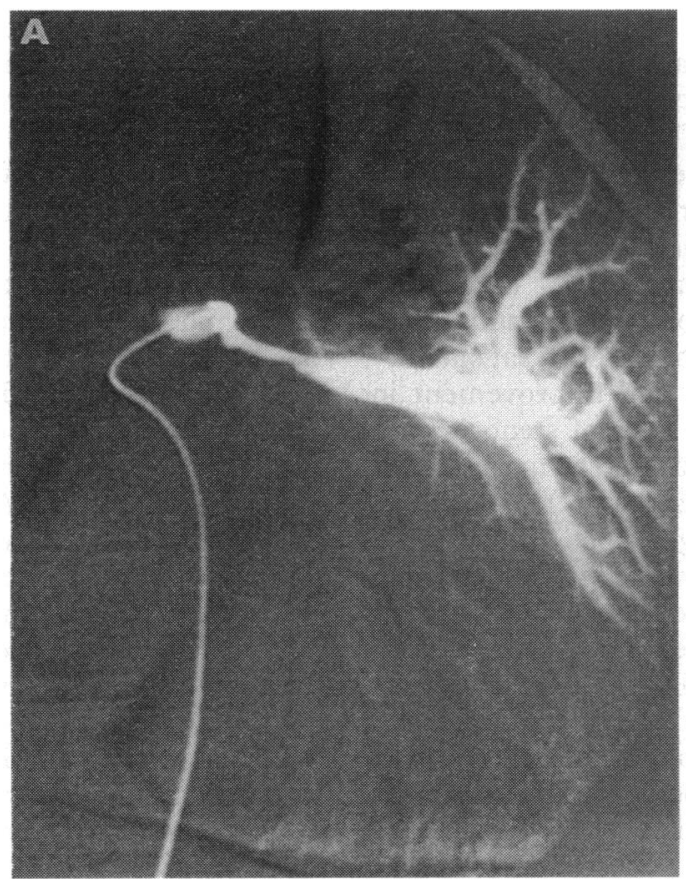

We report the case of a patient with complex pulmonary atresia who obtained symptomatic improvement and an increase in arterial oxygen saturation after implantation of balloon expandable stents into stenosed aortopulmonary collateral vessels.

\section{Case report}

A 22 year old man with pulmonary atresia, ventricular septal defect, and multiple aortopulmonary collateral vessels had developed severe cyanosis by the age of seventeen. A modified Pott's shunt (a gortex conduit from the descending aorta to the left pulmonary artery) was fashioned and the symptoms improved. Four years later he had become more cyanosed. The arterial oxygen saturation at rest was $78 \%$. At repeat cardiac catheterisation, no true central pulmonary arteries were identified and pulmonary blood supply was from multiple collaterals arising from the descending aorta. The modified Pott's shunt could not be identified. Selective catheterisation of the multiple collateral vessels with angiography showed pulmonary hypertension and pulmonary vascular disease

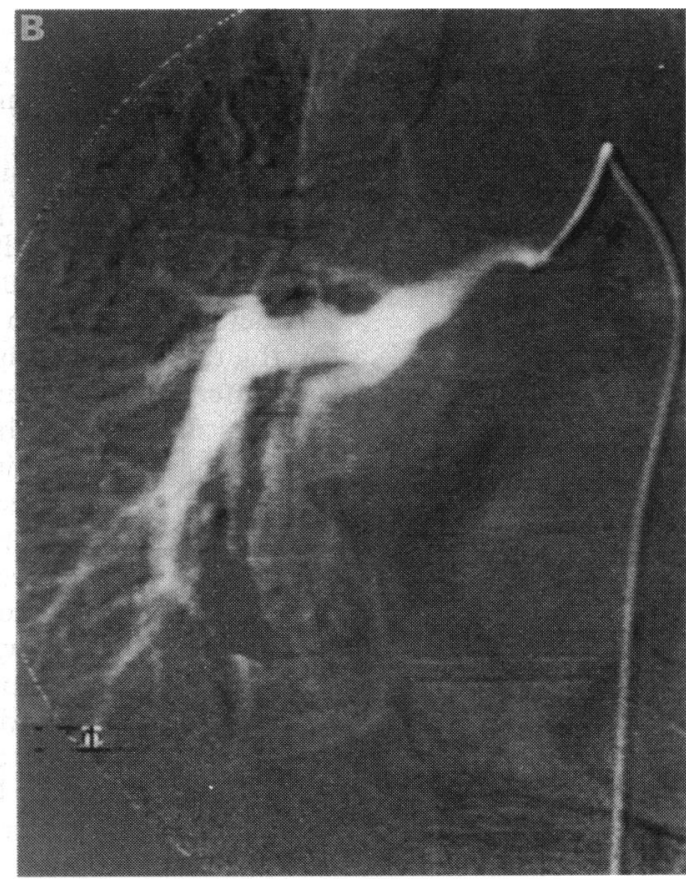

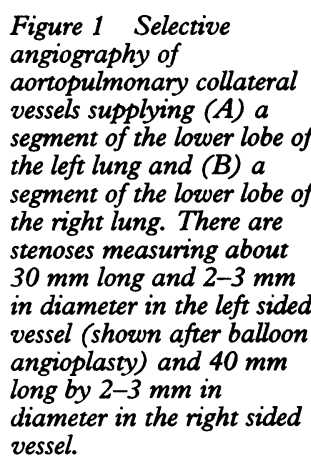



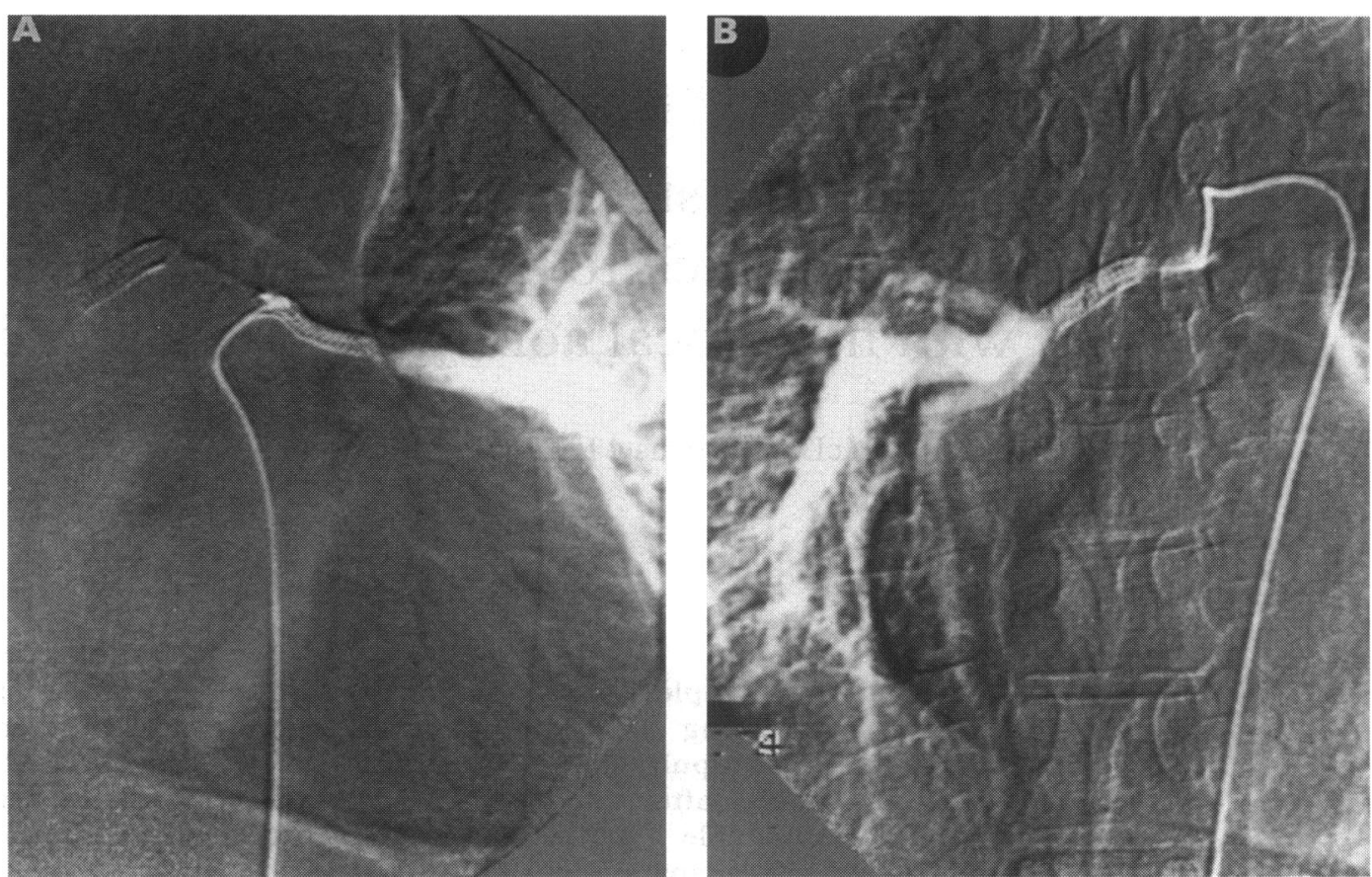

Figure 2 Selective angiography into the collateral vessels shown in fig 1 after stent implantation. The stents are clearly visible. Both stents are $40 \mathrm{~mm}$ long: their diameters are $4 \mathrm{~mm}$ on the left $(A)$, and $5 \mathrm{~mm}$ on the right $(B)$. There is a minor residual stenosis immediately distal to the stent on the left side and a similar minor stenosis proximal to the stent in the right sided vessel, with good flow to the distal segmental pulmonary arteries.

in the right upper lobe, with scant mediastinal collateral supply to a poorly perfused left upper lobe. There were stenoses in two large collateral vessels supplying segments of the right and left lower lobes (fig 1). The stenosis on the left measured $2-3 \mathrm{~mm}$ in diameter by $30 \mathrm{~mm}$ long and on the right, $2-3 \mathrm{~mm}$ in diameter by $40 \mathrm{~mm}$ long. The mean pressure distal to the stenosis on the right was $10 \mathrm{~mm}$ $\mathrm{Hg}$ and on the left was $20 \mathrm{~mm} \mathrm{Hg}$.

Balloon angioplasty of the left lower lobe collateral was performed with a $6 \mathrm{~mm}$ diameter balloon but the vessel recoiled to its initial size after balloon deflation. After unsuccessful attempts to pass a balloon mounted Strecker stent (Boston Scientific Ltd) down the collateral supplying the right lower lobe, the vessel was catheterised with an 8 French Mullins sheath that was advanced until the tip was distal to the stenosis. This allowed the stent to be positioned and uneventfully deployed at the required site. The procedure was repeated for the collateral supplying the left lower lobe. Both stents were $40 \mathrm{~mm}$ long, their final diameters were $4 \mathrm{~mm}$ on the left and $5 \mathrm{~mm}$ on the right. Angiography showed satisfactory placement of the stents and improvement in luminal diameter at the sites of stenoses, but with some residual minor narrowing immediately distal to the left sided stent and immediately proximal to the right sided stent (fig 2). The patient was treated with prophylactic cephradine and low dose aspirin. He was well until the next day when he developed left sided pleuritic chest pain and pyrexia. Chest radiography confirmed that the stents were in satisfactory positions and had not migrated, but there was some localised shadowing at the left lower lobe, consistent with either pulmonary infarction or infection. $\mathrm{He}$ was treated with anticoagulants and discharged eight days later on warfarin. At follow up two weeks later he reported subjective improvement in exercise tolerance and appeared less cyanosed. His resting arterial oxygen saturation had increased from $78 \%$ to $85 \%$.

\section{Discussion}

Balloon angioplasty has met with considerable success in improving pulmonary blood flow in a wide variety of congenital heart defects. ${ }^{2}$

In this patient balloon angioplasty alone was ineffective in relieving the stenoses, due to the elastic recoil of the vessel. Elastic recoil has been one of the main factors limiting success of balloon angioplasty in stenoses of branch pulmonary arteries but this problem has now been largely overcome by the introduction of balloon expandable stents. ${ }^{3}$ Stent implantation might, therefore, logically offer similar benefit in the treatment of stenosis of aortopulmonary collateral vessels.

Our choice of stent design was influenced by the tortuosity of the collateral vessels, the Strecker stent being flexible and able to conform to the natural curve of a vessel. The choice of the optimum stent diameter in this situation is difficult. A stent with too wide a diameter risks excessive pulmonary blood flow, whereas a device too small is unlikely to result in any real improvement and may even cause vessel obstruction. Our decision to use $4 \mathrm{~mm}$ and $5 \mathrm{~mm}$ diameter stents in the left and right sides respectively in this case was based upon the relatively small segments of lung supplied by the vessels in question. After our experience of stent implantation in other settings, ${ }^{4}$ we were optimistic that aspirin 
would be sufficient antithrombotic treatment in this patient, but the development of symptoms consistent with a possible pulmonary infarct led us to anticoagulate the patient with heparin and warfarin.

Stent implantation itself was uncomplicated and it is encouraging that the patient experienced subjective benefit and an improvement in arterial oxygen saturation at rest. This preliminary report suggests that stenting of stenosed aortopulmonary collateral vessels may provide a valuable alternative to surgical palliation in patients with complex pulmonary atresia.

1 Elzenga NJ. Pulmonary atresia. In: Hess J, Sutherland G eds. Congenital heart disease in adolescents and adults. Dordrecht: Kluwer Academic Publishers, 1990:103-15. 2 O'Laughlin MP, Perry SB, Lock JE, Mullins CE. Use of endovascular stents in congenital heart disease. Circulation 1991;83:1923-39.

3 Mullins CE, O'Laughlin MP, Vick GW III, et al. Implantation of balloon expandable intravascular grafts by catheterization in pulmonary arteries and systemic veins. Circulation 1988;77:188-99.

4 Gibbs JL, Rothman MT, Rees MR, Parsons JM, Blackburn ME, Carlos ER. Stenting of the arterial duct: a new approach to palliation for pulmonary atresia. $\mathrm{Br}$ Heart $\mathcal{f} 1992 ; 67: 240-5$.

\section{IMAGES IN CARDIOLOGY}

\section{An accidental aneurysm: an incidental finding}

\begin{abstract}
The magnetic resonance image shows a chronic traumatic thoracic aneurysm in a symptom free 30 year old woman. The aneurysm was almost certainly the consequence of a road traffic accident she had had 12 years before. It was picked up by routine chest radiography and its clear delineation by magnetic resonance imaging made invasive investigations unnecessary. The aneurysm was resected and found to be typical of those arising from a major deceleration injury.
\end{abstract}

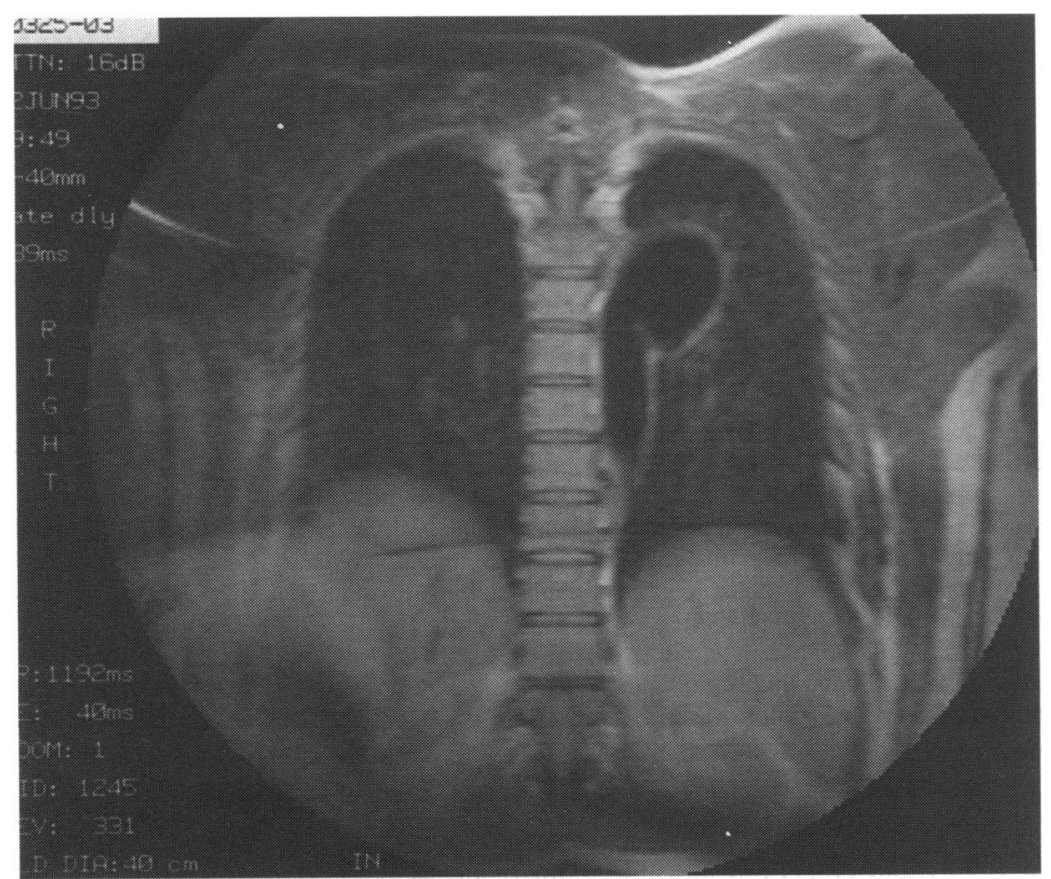

Magnetic resonance image (coronal spin-echo section; echo time $40 \mathrm{~ms}$ ) of the thorax delineating the thoracic aortic aneurysm.
There was an abrupt cut off margin where the aorta had been almost circumferentially transected. This woman was fortunate: she had undergone three uneventful pregnancies and normal deliveries since her accident.

In the sixteenth century Vesalius reported a chronic traumatic thoracic aneurysm in a man who had fallen from a horse. ${ }^{1}$ With the advent of the internal combustion engine high speed accidents increased and major deceleration injuries became more common.

The natural history of such aneurysms is difficult to assess accurately because undetected asymptomatic aneurysms cannot be included in the analyses. Finkelmeier et al reviewed reports of such aneurysms published between 1950 and $1980 .^{2}$ They found that $15 \%$ of a total of 390 cases had been managed conservatively. The remainder underwent surgical resection, with mortality of $4.6 \%$ and spinal damage in $1.4 \%$. None the less, long-term survival figures favoured a policy of surgical intervention: at 5 years survival was $93 \%$ in the surgically treated group and $71 \%$ in the observed group. This difference persisted 10 years after resection.

The fact that the wall of this aneurysm was fragile and thin 12 years after the accident accords with widely held belief that chronic traumatic thoracic aneurysms should not be assumed to have become stable after a prolonged period: once detected they should be resected.

$S$ A THORNE $S$ M FORBAT

1 Sailer S. Dissecting aneurysm of the aorta. Arch Pathol Lab Med 1942;33:704-30.

Finkelmeier BA, Mentzer RM, Kaiser DL, Tegtmeyer CJ Nolan SP. Chronic traumatic thoracic aneurysm. Thorac Cardiovasc Surg 1982;84:257-66. 